\title{
Quantum behaviour of hydrogen and muonium in vacancy-containing complexes in diamond
}

\author{
A Kerridge, A H Harker and A M Stoneham \\ Department of Physics and Astronomy, University College London, Gower Street, \\ London WC1E 6BT, UK \\ E-mail: a.kerridge@ucl.ac.uk
}

Received 7 September 2004, in final form 26 October 2004

Published 12 November 2004

Online at stacks.iop.org/JPhysCM/16/8743

doi:10.1088/0953-8984/16/47/024

\begin{abstract}
Most solid-state electronic structure calculations are based on quantum electrons and classical nuclei. These calculations either omit quantum zeropoint motion and tunnelling, or estimate it in an extra step. Such quantum effects are especially significant for light nuclei, such as the proton or its analogue, $\mu^{+}$. We propose a simple approach to including such quantum behaviour, in a form readily integrated with standard electronic structure calculations. This approach is demonstrated for a number of vacancy-containing defect complexes in diamond. Our results suggest that for the $\mathrm{NHV}^{-}$complex, quantum motion of the proton between three equivalent potential energy minima is sufficiently rapid to time-average measurements at X-band frequencies.
\end{abstract}

\section{Introduction: quantum behaviour of light nuclei}

Most solid-state electronic structure calculations are based on the Born-Oppenheimer approximation, in which rapidly moving quantum electrons follow the sluggish motion of heavy nuclei. The nuclear motion is often modelled in molecular dynamics using classical equations of motion. These calculations omit quantum zero-point motion and quantum tunnelling, effects that are most significant for light nuclei, such as the proton or its analogue, $\mu^{+}$. In the relatively rare cases that these quantum effects are considered, the approach adopted is usually a post hoc correction based on an adiabatic energy surface or, occasionally, a heavyweight calculation based on a Monte Carlo approach. In this paper, we propose a simple approach to including such quantum behaviour, in a form readily integrated with standard electronic structure calculations. The capability to handle quantum effects for hydrogen and hydrogen analogues is particularly important for semiconductor systems, in which $\mathrm{H}$ is of major importance because of its ubiquity, its mobility, and its ability to passivate electrically active impurities. We shall illustrate our approach for a defect system in diamond, partly because diamond has substantial similarities to conventional semiconductors, partly because diamond is showing increasing potential as 
an electronic material, and partly because there is a particular conflict between theory and experiment on which we might shed light.

\section{Approaches to predicting quantum behaviour}

Electronic structure calculations for static nuclei are very well established. Most state-ofthe-art calculations use some variant of a self-consistent field (SCF) method. The previous calculations that have explicitly included the quantum nature of the proton or muon (or an analogous hydrogen-like nucleus) are usually based on one of two methods.

(1) Post hoc SCF methods (e.g. [1, 2]), whereby an adiabatic potential energy surface is constructed from a series of SCF single-point energy calculations. We shall refer to these methods as PSCF. The wavefunction for the hydrogen-like nucleus is obtained using a numerical fit to this energy surface. These methods are time consuming, requiring a large number of SCF calculations for acceptable accuracy. This approach does not handle directly the effect of zero-point motion on atomic displacements, nor does it include fully the correlation energy between the electron and the hydrogen-like nucleus. More important, it is not clear that the adiabatic energy surface gives the right route to understanding systems in which tunnelling is important.

(2) Monte Carlo simulations [3, 4] that, in principle, could yield an exact solution to the many body Schrödinger equation, but are immensely computationally intensive, making calculations on large systems prohibitively expensive. It is not easy to use these methods to simulate more complicated processes, such as electron capture or emission events.

We describe here a third way to treat quantum effects. It works within the SCF cycle itself, and yields converged energies in times comparable to those required by standard electronic structure methods on systems of similar size. We model a single hydrogen-like nucleus using a basis set orthogonal to that of the electronic system. Put crudely, the hydrogen-like nucleus is treated like an electron with altered mass, charge and orthogonality relations. An analogous idea was used [5, 6] for defect complexes involving both electrons and holes in semiconductors. We have tested the approach, which we shall call HFQ1 (Hartree-Fock with quantum nucleus), on small organic molecules; further, we have validated the main features by comparison with simple, exactly soluble models. What we find is that the most simple approach overestimates the quantum effects, since its treatment of the correlation in the motions of the electrons and the hydrogen-like nucleus is not good enough. A simple generalization (HFQ2) yields a much better description, and we shall show that our approach resolves an apparent conflict between theory and experiment for a defect centre in diamond.

If all other nuclei are fixed, the total wavefunction of the system can be written as

$$
\Psi\left(\mathbf{r}_{\mathrm{e} 1}, \mathbf{r}_{\mathrm{e} 2}, \ldots, \mathbf{r}_{\mathrm{e} n}, \mathbf{r}_{\mu}\right)=\left|\psi_{1} \psi_{2} \cdots \psi_{n}\right| \psi_{\mu}\left(\mathbf{r}_{\mu}\right),
$$

where $\left|\psi_{1} \psi_{2} \cdots \psi_{n}\right|$ is an $n$-electron Slater determinant, and $\psi_{\mu}\left(\mathbf{r}_{\mu}\right)$ is the wavefunction of the hydrogen-like nucleus, formed as a linear combination of Gaussian basis functions. Our approach is readily extended to density functional methods. In principle, the method can be generalized to include configuration admixture, an approach considered by others [7].

The orthogonality of the electron basis set to that of the hydrogen-like nucleus leads to the Hamiltonian of the system having a block-diagonal form, allowing each block to be diagonalized separately, and yielding the $n$ lowest energy one-electron states in addition to the hydrogen-like nucleus ground state. The electron Hamiltonian matrix elements are modified from the standard Hartree-Fock form by the addition of a potential energy term corresponding 
Table 1. Comparison of zero-point energies and increases in bond lengths for hydrogen-like nucleus wavefunctions calculated using PSCF (reference case) and HFQ1.

\begin{tabular}{lllll}
\hline System & ZPEPSCF $(\mathrm{eV})$ & $\mathrm{ZPE}_{\mathrm{HFQ} 1}(\mathrm{eV})$ & $\Delta x_{\mathrm{PSCF}}(\AA)$ & $\Delta x_{\mathrm{HFQ} 1}(\AA)$ \\
\hline $\mathrm{C}_{2} \mathrm{H}_{5}$ & 0.17 & 0.57 & 0.017 & 0.000 \\
$\mathrm{C}_{2} \mathrm{H}_{4} \mathrm{Mu}$ & 0.47 & 1.52 & 0.052 & 0.000 \\
\hline
\end{tabular}

to the interaction of the electron density with the hydrogen-like nucleus. The hydrogen-like nucleus Hamiltonian matrix elements are given by

$$
H_{\lambda \sigma}^{(\mu)}=\left\langle\varphi_{\lambda}\left|\frac{-\nabla^{2}}{2 m_{\mu}}\right| \varphi_{\sigma}\right\rangle+\sum_{i}^{\text {ions }} Z_{i}\left(\varphi_{\lambda}\left|r_{i \mu}^{-1}\right| \varphi_{\sigma}\right)-V_{\lambda \sigma}^{\mathrm{e} \mu},
$$

where $r_{i \mu}=\left|\mathbf{r}_{i}-\mathbf{r}_{\mu}\right|$

$$
\left(\varphi_{\lambda}\left|r_{i \mu}^{-1}\right| \varphi_{\sigma}\right)=\int \varphi_{\lambda}^{*}\left(\mathbf{r}_{\mu}\right) r_{i \mu}^{-1} \varphi_{\sigma}\left(\mathbf{r}_{\mu}\right) \mathrm{d} \mathbf{r}_{\mu},
$$

and, using the HFQ1 approach, $V_{\lambda \sigma}^{\mathrm{e} \mu}$ is given by

$$
\begin{aligned}
V_{\lambda \sigma}^{\mathrm{e} \mu} & =\sum_{\eta, \nu}^{\text {elec. }} P_{\eta \nu}^{\mathrm{e}}\left(\varphi_{\lambda} \varphi_{\sigma} \mid \varphi_{\eta} \varphi_{\nu}\right) \\
& =\sum_{\eta, \nu}^{\text {elec. }} P_{\eta \nu}^{\mathrm{e}} \iint \varphi_{\lambda}^{*}\left(\mathbf{r}_{\mu}\right) \varphi_{\sigma}\left(\mathbf{r}_{\mu}\right) r_{\mathrm{e} \mu}^{-1} \varphi_{\eta}^{*}\left(\mathbf{r}_{\mathrm{e}}\right) \varphi_{\nu}\left(\mathbf{r}_{\mathrm{e}}\right) \mathrm{d} \mathbf{r}_{\mu} \mathrm{d} \mathbf{r}_{\mathrm{e}} .
\end{aligned}
$$

We illustrate the quantum effects, notably zero-point motion, by considering the wavefunction of a hydrogen-like nucleus in the ethyl radical, $\mathrm{C}_{2} \mathrm{H}_{5}$, and its muonated analogue, $\mathrm{C}_{2} \mathrm{H}_{4} \mathrm{Mu}$. Reference wavefunctions were calculated using a post hoc SCF method, as described above, and compared to those calculated using the HFQ1 approach with a quantum hydrogenlike nucleus, obtained by the diagonalization of the Hamiltonian described by equation (2). The hydrogen-like nucleus ( $\mathrm{p}$ or $\mu^{+}$) basis set used in these calculations (and throughout this paper) consisted of a set of six Gaussians with exponents ranging from 0.4 to 25 centred on two sites along the $\mathrm{C}-\mathrm{X}(\mathrm{X}=\mathrm{H}, \mathrm{Mu})$ bond, which was found to yield energies converged to $0.01 \mathrm{eV}$ [8]. The electron basis set was a standard 6-31G set, which suffices for the points to be demonstrated. All calculations were performed using Gaussian 98 [9], modified to include the quantum hydrogen-like nucleus.

In particular, we examined the effect of our HFQ1 approximations on the zero-point energy of the hydrogen-like nucleus, calculated as the expectation value of the kinetic energy operator with respect to the ground state. This would reveal any overlocalization of the hydrogen-like nucleus wavefunction. Further, we studied the increase of the average bond length over the classical value, $\Delta x=\langle\psi|x| \psi\rangle$, which reveals the accuracy to which bond anharmonicity is modelled. Table 1 shows the results of these calculations.

As can be seen, the HFQ1 approximation severely overlocalizes the wavefunction of the hydrogen-like nucleus, hence increasing the zero-point energy by a factor of approximately three. The HFQ1 also predicts a wavefunction symmetric about the classical potential energy minimum, in contrast to the reference calculations, which predict a mass-dependent increase in the equilibrium bond-length. The same features from the HFQ1 method were observed over a range of systems, where the hydrogen-like nucleus was bonded to oxygen, nitrogen, oxygen, phosphorus, and sulfur [8]. Furthermore, the overlocalization of the wavefunction was also in evidence when the Hartree-Fock approximation was imposed on a model system of harmonically coupled particles of differing mass, for which the correlation energy and exact two-particle wavefunction could be evaluated analytically [10]. 


\subsection{Improving the correlation of the hydrogen-like nucleus with electrons}

In the Born-Oppenheimer approximation, electrons follow the nuclear motion adiabatically. Our HFQ1 approximation groups the hydrogen-like nucleus with the electrons, ensuring that the proton or muon also follows the motion of the heavier atoms adiabatically. But, so far as the electrons are concerned, even the muon is a heavy particle. This is well known, of course, and is the basis of the assumptions in the quantum theory of hydrogen diffusion [11].

Our present approach is an alternative to the multiconfigurational SCF method of [7], which showed that their single-configuration Hartree-Fock wavefunctions were not suitable for an accurate description of effects such as tunnelling through neglect of dynamical correlation. We adopt an alternative method to model this correlation through the use of a parametrized potential, obtaining a post-Hartree-Fock description of the hydrogen-like nucleus. There are some parallels with the approach of Spaeth and Seidel [12] (see also [13, p 683]). We find that, for an analytically soluble system, these correlation effects are much more pronounced in the nuclear component of the wavefunction than in the electronic component $[8,10]$.

We now assess this simple extension of the ideas of the HFQ1 approach to model the correlation in the motions of the hydrogen-like nucleus and the electrons. In this extension, HFQ2, we approximate the true interaction with a parametrized potential, $V^{\mathrm{p}}$, defined as

$$
V^{\mathrm{p}}=f_{\mathrm{c}} V^{\mathrm{BO}}+\left(1-f_{\mathrm{c}}\right) V^{\mathrm{HF}},
$$

where typical matrix elements of $V^{\mathrm{BO}}$ and $V^{\mathrm{HF}}$ are given by

$$
\begin{aligned}
V_{\lambda \sigma}^{\mathrm{HF}} & =-\sum_{\eta, \nu} P_{\eta \nu}\left(\varphi_{\lambda} \varphi_{\sigma} \mid \varphi_{\eta} \varphi_{\nu}\right) \\
V_{\lambda \sigma}^{\mathrm{BO}} & =-\sum_{\eta, \nu} P_{\eta \nu} S_{\lambda \sigma}\left(\varphi_{\lambda}\left|r_{\mathrm{e} \mu}^{-1}\right| \varphi_{\sigma}\right) .
\end{aligned}
$$

Here $S_{\lambda \sigma}$ is an overlap matrix element, and $V^{\mathrm{p}}$ replaces $V^{\mathrm{e} \mu}$ in equation (2). Note that, in equation $(6 b), \mathbf{r}_{\mu}$ represents the position at which the basis function is centred, and is not integrated over. For a correlation factor $f_{\mathrm{c}}=0$, we regain our approximation HFQ1. As $f_{\mathrm{c}}$ tends to unity, we regain the usual adiabatic approximation. In practice however, we find a very badly behaved matrix in this limit due to the similarity in different matrix elements of the Hamiltonian, and since the limit is far from the optimum value of $f_{\mathrm{c}}$, we have not studied it in detail.

The new $V^{\mathrm{p}}$ is found [8] to be able to model accurately the correlated hydrogen-like nucleus-electron interaction, agreeing well with results using a PSCF method. The correlation factor, $f_{\mathrm{c}}$, of equation (5) proves to show well defined and systematic behaviour with the mass of the hydrogen-like nucleus, being proportional to $m_{\mu}^{-1 / 2}$. We shall show later that $f_{\mathrm{c}}$ seems respectably transferable from one system to another. So, whilst there is an empirical input, the approach is by no means arbitrary. Table 2 shows that the HFQ2 model reproduces the results of the reference calculations (table 1) much more effectively than does HFQ2, especially for the case of the lighter muon. In section 3, we shall take the HFQ2 model further, applying it to vacancy-containing complexes in diamond.

\section{Vacancy complexes in diamond: results using the HFQ2 extended model}

Hydrogen is ubiquitous in diamond, where concentrations of up to 1 at.\% have been reported $[14,15]$. H passivates both donors and acceptors in diamond [16], removing the electronically active levels from the bandgap. Goss [16] gives a review of the theoretical work in this area. Hydrogen can be hard to detect experimentally, partly because of the strong tendency for interstitial atomic hydrogen to form molecules [17]. In consequence, 

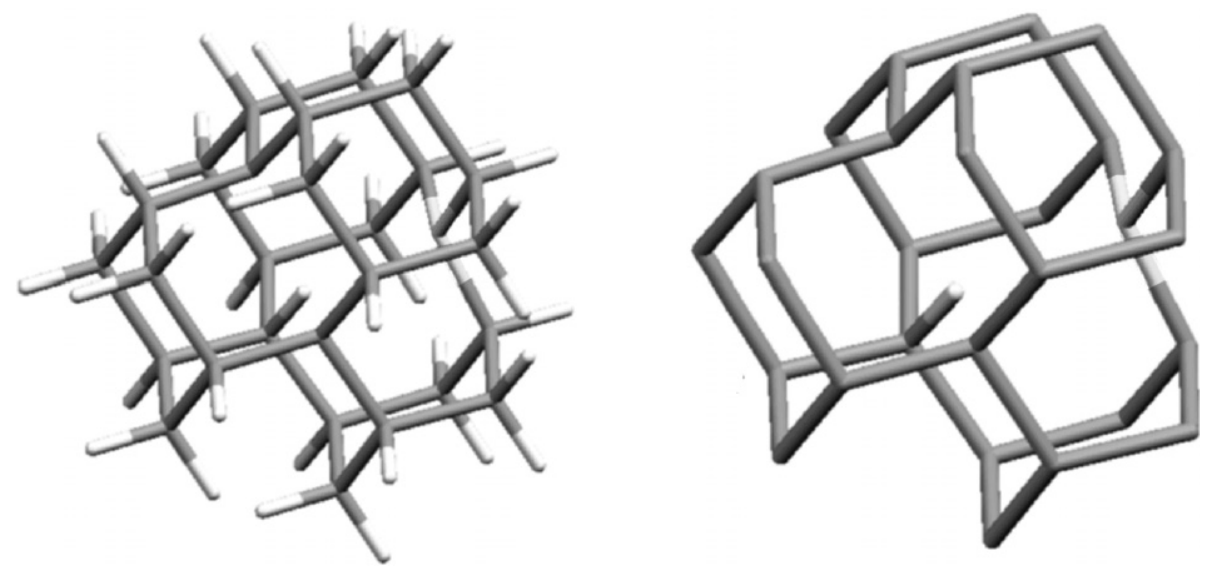

Figure 1. The $\mathrm{C}_{27} \mathrm{H}_{36}$ IX vacancy-centred cluster used for all calculations (I is $\mathrm{C}, \mathrm{N}$. P or S). The left-hand figure shows the bond-saturating hydrogens at the outer surface.

Table 2. Results for $\mathrm{C}_{2} \mathrm{H}_{5}$ and $\mathrm{C}_{2} \mathrm{H}_{4} \mathrm{Mu}$ using the parametrized potential of equation (5). The HFQ2 model reproduces the results of the reference calculations (table 1) far more effectively than HFQ1, particularly in the case of the lighter muon.

\begin{tabular}{llllll}
\hline System & $f_{\mathrm{c}}$ & ZPEPSCF $(\mathrm{eV})$ & $\mathrm{ZPE}_{\mathrm{HFQ} 2}(\mathrm{eV})$ & $\Delta x_{\mathrm{PSCF}}(\AA)$ & $\Delta x_{\mathrm{HFQ} 2}(\AA)$ \\
\hline $\mathrm{C}_{2} \mathrm{H}_{5}$ & 0.758 & 0.17 & 0.17 & 0.017 & 0.009 \\
$\mathrm{C}_{2} \mathrm{H}_{4} \mathrm{Mu}$ & 0.667 & 0.47 & 0.50 & 0.051 & 0.052 \\
\hline
\end{tabular}

many of the experimental results have been obtained from muon spin experiments ( $\mu$ SR; for a review, see $[18,19])$, where a positively charged muon $\mu^{+}$, sometimes with its trapped electron $\left(\mathrm{Mu}^{0}=\left[\mu^{+} \mathrm{e}\right]\right)$, is an analogue to hydrogen. The muon, however, is much lighter than the proton $\left(m_{\mu} / m_{\mathrm{p}} \approx 1 / 9\right)$, and so consequently any quantum mechanical features of its interactions will be larger than those of the proton. Our paper is concerned with the extent to which the analogy between muons and protons can be quantified. We analyse this using the HFQ2 model, which uses the parametrized model potential to calculate the correlated interactions of the proton (muon). Specifically, we discuss H or Mu trapped at an impurityvacancy complex in diamond in which $\mathrm{N}, \mathrm{S}$, or $\mathrm{P}$ is a neighbour to the vacancy.

We have applied the parametrized model of section 2 to four systems, namely the $\mathrm{XV}$, NXV, PXV, and SXV (where X $=\mathrm{H}, \mathrm{Mu}$ ) defect complexes. Gaussian 98 was used for all calculations, with hydrogen-like nucleus and electron basis sets as described in section 2 . The model system was a hydrogen-terminated diamond cluster $\mathrm{C}_{27} \mathrm{H}_{36} \mathrm{IX}$, where $\mathrm{I}=\{\mathrm{C}, \mathrm{N}, \mathrm{P}, \mathrm{S}\}$, centred on a vacancy (see figure 1). Such a small cluster is acceptable only for the assessment of $\mathrm{H}$ or $\mathrm{Mu}$ quantum effects so, for other aspects, where appropriate, we shall draw on published calculations by others. For the purposes of relaxation, the hydrogen-like nucleus was replaced by a classical point charge, with any inaccuracies introduced by this expected to be negligible [20]. Symmetric relaxation of nearest and next nearest neighbour carbons, as well as the impurity and hydrogen-like particle, was allowed. In all cases the classical $\mathrm{H}$ was found to bind to the unsaturated carbon.

Table 3 shows the bond-length increases and zero-point energies of the hydrogen-like nucleus in these defect complexes, calculated using the HFQ2 model, along with comparisons to reference calculations, obtained using the PSCF model. The correlation parameters $f_{\mathrm{c}}$ used were those obtained for the $\mathrm{C}-\mathrm{X}$ bond in a group of small organic molecules [8], 
Table 3. Bond-length increases and zero-point energies of the hydrogen-like nucleus, calculated using the parametrized potential of equation (5). Reference values were obtained using the PSCF model.

\begin{tabular}{llllll}
\hline Complex & $f_{\mathrm{c}}$ & $\mathrm{ZPE}_{\mathrm{PSCF}}(\mathrm{eV})$ & $\mathrm{ZPE}_{\mathrm{HFQ} 2}(\mathrm{eV})$ & $\Delta x_{\mathrm{PSCF}}(\AA)$ & $\Delta x_{\mathrm{HFQ} 2}(\AA)$ \\
\hline $\mathrm{MuV}$ & 0.663 & 0.57 & 0.57 & 0.048 & 0.046 \\
$\mathrm{NMuV}$ & 0.663 & 0.60 & 0.61 & 0.044 & 0.042 \\
$\mathrm{PMuV}$ & 0.663 & 0.59 & 0.63 & 0.043 & 0.040 \\
$\mathrm{SMuV}$ & 0.663 & 0.63 & 0.62 & 0.051 & 0.041 \\
$\mathrm{HV}$ & 0.758 & 0.19 & 0.24 & 0.016 & 0.004 \\
NHV & 0.758 & 0.20 & 0.26 & 0.014 & 0.003 \\
PHV & 0.758 & 0.20 & 0.26 & 0.011 & 0.003 \\
SHV & 0.758 & 0.22 & 0.26 & 0.011 & 0.003 \\
\hline
\end{tabular}

Table 4. Changes in defect formation energy due to correlation of the hydrogen-like nucleus and electron. The extra significant figures have been retained to show the weak trends.

\begin{tabular}{lll}
\hline Complex & $\Delta E_{\mathrm{D}}^{\mathrm{p}}(\mathrm{eV})$ & $\Delta E_{\mathrm{D}}^{\mu}(\mathrm{eV})$ \\
\hline $\mathrm{XV}$ & 0.58 & 1.71 \\
$\mathrm{NXV}$ & 0.58 & 1.76 \\
PXV & 0.59 & 1.75 \\
SXV & 0.60 & 1.78 \\
\hline
\end{tabular}

although it should be noted that the local environment in the systems considered here is significantly different from the molecular case, with some localization of the hydrogen-like nucleus wavefunction expected due to repulsion from the surrounding lattice.

As expected, there is an increase in the zero-point energies in these systems as hydrogen is replaced by muonium, although there is only a small corresponding change in the bond-length increase. This suggests that the increases in zero-point energy are due to a localization across the bond, i.e., that the stretch mode frequencies remain largely unaffected. Most notable is that the PSCF reference calculations and our HFQ2 calculations agree well using the parameters obtained for molecular systems. It seems that the parameter $f_{\mathrm{c}}$ depends predominately on the bond type, and not on the environment at large. It is possible, of course, to improve the results by choosing an optimum value of $f_{\mathrm{c}}$ for each system, but this would obscure the relative simplicity and transferability that we have established.

Although the results presented in table 3 illustrate how the hydrogen-like nucleus wavefunction differs in the solid-state and molecular environment, it is more interesting to consider how the hydrogen-like nucleus-electron correlation energy affects the defect formation energy. The absolute formation energies have not been calculated here (see [21]), but the relative formation energies given here can be used to consider how those energies calculated classically must be modified when taking quantum effects into account. The defect formation energies, relative to the classical defect formation energy $E_{\mathrm{D}}^{\mathrm{c}}$, are given in table 4 .

These changes in energies are some three times larger than the corresponding zero-point energies, and illustrate the fact that post hoc methods, although able to model the hydrogenlike nucleus wavefunction accurately, are unreliable when considering properties which are dependent on total energies.

\section{Intersite quantum tunnelling and quantum diffusion}

Recent experimental work [22] on the $\mathrm{NHV}^{-}$defect complex in diamond indicates that the complex has $\mathrm{C}_{3 \mathrm{v}}$ symmetry. Its [111] symmetry direction seems to suggest that the $\mathrm{H}$ bonds 
to $\mathrm{N}$, and not to one of the three unsaturated carbons surrounding the vacancy. This result would contradict theoretical calculations in which $\mathrm{H}$ is treated classically [21]. It has been suggested [21] that the experimentally observed symmetry could be explained by rapid motion of $\mathrm{H}$ around the three equivalent $\mathrm{C}$ sites. Since the experimental symmetry is observable at a temperature of $4.2 \mathrm{~K}$, this would suggest that (coherent) quantum tunnelling or an incoherent quantum diffusion mechanism may be responsible for the seeming disparity between experiment and theory. We present here a zeroth order calculation of the coherent tunnelling rate of $\mathrm{H}$ and $\mathrm{Mu}$ about these three centres. The results we give are for both the neutral and negatively charged states of the NHV complex.

In calculating the intersite tunnel rate, we follow the approach of Herring [23] that only requires knowledge of the localized hydrogen-like nucleus wavefunction in a single well, as calculated in section 3 . This method uses symmetry-adapted linear combinations of these single-well wavefunctions, in analogy to those given by equation (5) of [7], and gives a measure of the splitting between the delocalized ground state and the corresponding excited state. There is an implicit assumption that the ground and excited states only differ in the nuclear component of the many body wavefunction, an assumption justified for two reasons. First, the nuclear component is calculated via the parametrized potential of equation (5) that is designed to include the effects of dynamical correlation between the nucleus and electrons, so giving an accurate representation of this component of the wavefunction. Secondly, the overlap between the nuclear wavefunctions in different potential minima is small, and so the corresponding nuclear charge density is almost identical for the ground and excited states.

Herring's original discussion was concerned with the exchange splitting in $\mathrm{H}_{2}^{+}$, a doublewell system, but the general method presented in [23] can be adapted to obtain the tunnel splitting energy of a particle in a symmetric two-well system. In this system a basis set consisting of the symmetric and antisymmetric combinations of the two single-well wavefunctions $\varphi_{a}$ and $\varphi_{b}$ is used in order to deduce the value of the tunnel-splitting energy, given by

$$
\Delta E=2 J=2\left\langle\varphi_{\mathrm{a}}|\hat{H}| \varphi_{\mathrm{b}}\right\rangle
$$

hence estimating the tunnelling rate [24].

The NHV complex can be considered in an analogous way, with the three equivalent potential energy minima located on the vertices of an equilateral triangle. In this case, the $C_{3 v}$ symmetry-adapted nuclear basis for the calculation is given by the functions

$$
\begin{aligned}
& \psi_{1}=\frac{1}{\sqrt{3}}\left(\varphi_{\mathrm{a}}+\varphi_{\mathrm{b}}+\varphi_{\mathrm{c}}\right) \\
& \psi_{2}=\frac{1}{\sqrt{2}}\left(\varphi_{\mathrm{a}}-\varphi_{\mathrm{b}}\right) \\
& \psi_{3}=\frac{1}{\sqrt{6}}\left(\varphi_{\mathrm{a}}+\varphi_{\mathrm{b}}-2 \varphi_{\mathrm{c}}\right) .
\end{aligned}
$$

Here $\psi_{1}$ is the ground state with energy $E_{+}=E_{0}+2 J$, whilst $\psi_{2}$ and $\psi_{3}$ are degenerate with energy $E_{-}=E_{0}-J$. This gives a tunnel-splitting energy $\Delta E=3 J$, which can be evaluated explicitly as [8]

$$
\Delta E=\frac{3}{m} \iint \varphi_{\mathrm{a}}(0, y, z) \frac{\partial \varphi_{\mathrm{a}}(0, y, z)}{\partial x} \mathrm{~d} y \mathrm{~d} z,
$$

where $\varphi_{\mathrm{a}}(0, y, z)$ indicates that $\varphi_{a}$ should be evaluated at $x=0$, and similarly for its derivative. This generalization of Herring's approach to the three-well system does not appear to have been given before. 
Table 5. Tunnel splitting energies and tunnelling frequencies of $\mathrm{H}$ and $\mathrm{Mu}$ in vacancy-containing complexes in diamond $(\mathrm{X}=\mathrm{N}, \mathrm{S}, \mathrm{P})$. We have kept enough significant figures to make weak trends visible.

\begin{tabular}{lllll}
\hline Complex & $\Delta E^{\mathrm{p}}\left(\mathrm{cm}^{-1}\right)$ & $\tau_{\mathrm{p}}\left(\times 10^{-12} \mathrm{~s}\right)$ & $\Delta E^{\mu}\left(\mathrm{cm}^{-1}\right)$ & $\tau_{\mu}\left(\times 10^{-12} \mathrm{~s}\right)$ \\
\hline NXV & 5.1 & 6.6 & 150 & 0.23 \\
PXV & 4.2 & 7.9 & 160 & 0.21 \\
SXV & 6.4 & 5.2 & 290 & 0.12 \\
\hline
\end{tabular}

Table 6. Zero-point energies, tunnel splitting energies and tunnelling frequencies of $\mathrm{H}$ and $\mathrm{Mu}$ in the $\mathrm{NXV}^{-}$complex.

\begin{tabular}{llcl}
\hline Complex & $\mathrm{ZPE}_{\mathrm{HFQ} 2}(\mathrm{eV})$ & $\Delta E\left(\mathrm{~cm}^{-1}\right)$ & $\tau\left(\times 10^{-12} \mathrm{~s}\right)$ \\
\hline $\mathrm{NHV}$ & 0.21 & 4.1 & 8.1 \\
$\mathrm{NMuV}$ & 0.60 & 160 & 0.21 \\
\hline
\end{tabular}

Unlike the double-well system, where the time evolution of the system is such that the hydrogen-like nucleus localizes in each well in turn, in the triple-well system a particle localized in a given well never completely localizes in another well, but continues to relocalize in the original well with period $\tau=2 \pi / \Delta E$. Table 5 gives the tunnel-splitting energies and tunnelling frequencies of the hydrogen-like nucleus in the NXV, SXV, and PXV complexes.

The experimental evidence for the trigonal [111] symmetry of the $\mathrm{NHV}^{-}$centre is based on X-band electron spin resonance [22], a technique with a characteristic time resolution of $\sim 10^{-10} \mathrm{~s}$. Our results for $\mathrm{NHV}^{0}$ predict that hydrogen would tunnel sufficiently rapidly that the time-averaged result should be obtained at typical spin resonance frequencies: even the slowest tunnel rate predicted is more than an order of magnitude larger than the characteristic time of ESR. We have also performed HFQ2 calculations on the negatively charged centre $\mathrm{NHV}^{-}$using the parameters given in table 3, the results of which can be seen in table 6 .

The first point to notice here is the fact that the properties of the hydrogen-like nucleus remain largely unaffected by the change in charge state, implying that the electron density local to the complex remains reasonably constant, although there might be a significant change in spin density. The similarity of the effective potential energy surface on which the hydrogenlike nucleus moves for each charge state was verified using PSCF. The similarity in tunnel rates for $\mathrm{NHV}^{0}$ and $\mathrm{NHV}^{-}$suggests that $\mathrm{X}$-band ESR should show $\mathrm{H}$ delocalized over the three equivalent carbon centres, giving the observed symmetry, $\mathrm{C}_{3 \mathrm{v}}$. The quantum effects we discuss are essential to give this agreement with experiment, for Goss et al [21] find a barrier for $\mathrm{H}$ motion between these $\mathrm{C}$ centres of $0.4 \mathrm{eV}$, significantly higher than the proton zero-point energy. At low temperatures, at least, classical diffusion of the proton can be discounted, even if post hoc zero-point energy is included. We note that, if it were possible to measure the symmetry of $\mathrm{NHV}^{0}$ or $\mathrm{NHV}^{-}$by an optical experiment (for which the characteristic time might be femtoseconds), then the lower symmetry should be seen, with $\mathrm{H}$ apparently localized on just one of the carbons.

\section{Conclusions}

We have presented an efficient approach to model some of the key quantum features of hydrogen-like nuclei. The basic approach HFQ1, in which the hydrogen-like nucleus is handled in a way similar to the treatment of electrons, is extended to give a parametrized model, HFQ2, for which the parameters appear to be respectably transferable. This HFQ2 
model appears to work well in predicting the quantum behaviour of hydrogen and muonium in vacancy-containing defect complexes in diamond. Our results suggest a reconciliation of previous disagreements between experiment and theory, and highlight the weaknesses of the standard Hartree-Fock approximation in predicting such quantum effects. In particular, post hoc SCF methods are insufficient for predicting properties of the system dependent on the total energy, since the hydrogen-like nucleus-electron correlation energy cannot be included implicitly using such methods.

We find that, for the $\mathrm{NHV}^{-}$complex in diamond, quantum motion of the proton is sufficiently rapid that electron spin resonance, as used in [22], would show a time-averaged signal. We conclude that quantum diffusion is a viable mechanism for reconciling the apparently differing experimental and theoretical results for this system. This will be discussed in detail in a separate paper.

\section{Acknowledgments}

We wish to thank Professor S J F Cox for helpful discussions, and the Rutherford Appleton Laboratories and EPSRC for financial support.

\section{References}

[1] Boxwell M A et al 1993 J. Chem. Soc. Faraday Trans. 892957

[2] Claxton T A et al 1990 Hyperfine Interact. 65913

[3] Ramirez R and Hererro C P 1994 Phys. Rev. Lett. 73126

[4] Miyake T et al 1998 Phys. Rev. Lett. 811873

[5] Stoneham A M and Harker A H 1975 J. Phys. C: Solid State Phys. 81102

[6] Stoneham A M and Harker A H 1975 J. Phys. C: Solid State Phys. 81109

[7] Pak M V and Hammes-Schiffer S 2004 Phys. Rev. Lett. 92103002

[8] Kerridge A 2003 PhD Thesis University College London

[9] Frisch M J et al 1998 Gaussian 98 Revision A.7 (Pittsburgh, PA: Gaussian)

[10] Kerridge A et al 2005 at press

[11] Flynn C P and Stoneham A M 1970 Phys. Rev. B 13966

[12] Spaeth J-M and Seidel H 1971 Phys. Status Solidi 46323

[13] Stoneham A M 1975 Theory of Defects in Solids (Oxford: Oxford University Press)

[14] Dischler B et al 1993 Physica B 185217

[15] Sellschop J P F et al 1977 Ind. Diamond Rev. Suppl. 2-4

[16] Goss J P 2003 J. Phys.: Condens. Matter 15 R551

[17] Mainwood A and Stoneham A M 1984 J. Phys. C: Solid State Phys. 172513

[18] Cox S F J 1986 J. Phys. C: Solid State Phys. 203187

[19] Cox S F J 2003 J. Phys.: Condens. Matter 15 R1727

[20] Porter A R et al 1999 Phys. Rev. B 6013534

[21] Goss et al 2003 J. Phys.: Condens. Matter 15 S2903

[22] Glover C et al 2003 Phys. Rev. Lett. 90185507

[23] Herring C and Flicker M 1964 Phys. Rev. 134 A362

[24] Merzbacher E 1998 Quantum Mechanics 3rd edn (Chichester: Wiley) 